\title{
Structural Health Monitoring by Magnetic Sensing in Concrete Structures via Embedded Shape Memory Alloy Components
}

\author{
Allen Davis, Mirmilad Mirsayar, and Darren Hartl* \\ Aerospace Engineering Department, Texas A\&M University, College Station, Texas
}

\begin{abstract}
In the field of structural health monitoring (SHM), innovative methods of non-destructive evaluation (NDE) are currently being investigated with the purpose of providing prognostic information toward safer, longer lasting structures. Therefore, it is desirable to integrate NDE techniques with existing structural reinforcement techniques for in situ measurement capability, increasing service life. Magnetic shape memory alloys (MSMAs) offer the potential for NDE via magnetic sensing, while further offering the multi-functionality of crack closing and structural reinforcement. The current research proposes a novel SHM approach for concrete structures using embedded MSMAs for magnetic sensing, and investigates the properties of such a system.
\end{abstract}

\section{Introduction}

Cracks are formed and propagate in concrete structures due to applied mechanical or environmental loads leading to catastrophic failure in infrastructures. Along with advancements in understanding of fracture properties of concrete materials [1-3], the structural health monitoring (SHM) of concrete structures has also received significant attention by the researchers by developing advanced methods in non-destructive evaluation. A relatively new field of exploration for SHM is non-destructive evaluation via magnetic sensing of embedded secondary materials, evaluating the external magnetic fields around structural components. Magnetic shape memory alloys (MSMAs), known for their ability to change magnetic and other properties given solid to solid phase change, can alter magnetic fields due to their change in permeability in the presence of internal stress. Magnetic sensing may provide an advantage for SHM in that the magnetic permeability of concrete, water, and air are nearly identical to that of free space and have no noticeable effect on the magnetic field generated by a permanent magnet; therefore, all sensing changes can be attributed to localized stress induced phase changes in the embedded MSMA. This use of magnetic sensing for non-destructive evaluation represents an inherently multidisciplinary research area with respect to both the computational and experimental domain.

Fortunately, a history of previous successes for each method exists individually. Shape memory alloy (SMA) wires have been embedded into concrete specimens for structural reinforcement and shown to improve the life of the structure [4], taking advantage of the super-elastic properties of MSMAs. Ferromagnetic shape memory alloys, specifically FeMnAlNi (FMAN), have been developed with the strength required for structural reinforcement and a recoverable super elastic effect [5], and samples of these specimens have been produced by Karaman and coworkers [6]. Embedded inductive sensors have been developed to monitor the corrosion of internal steel rebar [7], but this technique requires an applied electric current, and only shows the average corrosion between two points. Additional work on monitoring steel corrosion has proven magnetic sensing a viable method of non-destructive evaluation of similar structures, with obtained magnetic signals shown to be significantly strong as compared the background noise present in the system [8]. That work also proposed Giant Magneto-Resistive (GMR) sensors, which measure magnetic field strength, as a possible magnetic sensor for non-destructive evaluation of internal steel bars due to low power consumption and a wide sensing range. As for interpreting the detected magnetic fields, computational work has been previously done by Hartl and coworkers to transform signals generated from distributions of distinct embedded MSMA domains into internal continuous stress fields [9]. Embedding shape memory alloy (SMA) wires in concrete components offer the potential to monitor their structural health via external magnetic field sensing. SMAs have been used to close internal cracks, structurally reinforce concrete structures, and reduce fatigue under cyclic loading [10, 11], so the addition of such a multi-functional material for the purpose of structural evaluation is very desirable. Thus, an evaluation of SMAs for magnetic sensing is required for both the structural and magnetic domains.

\section{Modeling description}

Structural health monitoring using magnetic sensing for non-destructive evaluation requires evaluating the

\footnotetext{
* Corresponding author: darren.hartl@tamu.edu
} 
change in an external magnetic field to determine the health of the internal structure. For this change to have a significant meaning, the magnetic field change must be correlated to specific internal flaws or damage incurred. This requires a robust computational framework to evaluate both the structural mechanics of internal damage, the response of an embedded MSMA, and the magnetic response of the system due to the change in magnetization of the MSMA.

Due to the different constitutive responses required for evaluation, the computational framework is divided into two distinct models: a structural mechanics model using finite element methods to evaluate the mechanical response of specified structural damage on an embedded MSMA, and a magneto-static model to evaluate the sensing capabilities of an external magnet and sensor combination in detecting the structural damage using the magneto-mechanical response of the MSMA.

\subsection{Structural modeling}

A computational model is needed to predict the change in martensite volume fraction in the MSMA wire near a crack under high stress. This model is required to predict the material fracture properties near a crack tip, and the subsequent change in wire phase due to the crack tip field.

The structural mechanics of the concrete-MSMA wire system are modeled in Abaqus, a commercial finite element software, using extended finite element method (XFEM) to obtain the fracture behavior of the concrete near the crack tip. The model consists of an embedded wire in a concrete block with a pre-existing crack under a three-point bend loading condition. The concrete specimen is a $150 \mathrm{~mm}$ by $50 \mathrm{~mm}$ by $50 \mathrm{~mm}$ block, the same size as the samples used for structural testing, with the load frame supports under the block $150 \mathrm{~mm}$ apart. The wire has a $1 \mathrm{~mm}$ radius and is embedded $15 \mathrm{~mm}$ away from the bottom face of the concrete block. The model applies a displacement at the center of the top face of the concrete block as shown in Figure 1, inducing crack propagation and internal structural damage. The crack located in the concrete specimen opposite the loading propagates toward the MSMA wire, creating a local stress concentration near the crack tip. As this crack tip stress field approaches the MSMA wire, the wire transforms locally near the internal damage in the concrete.

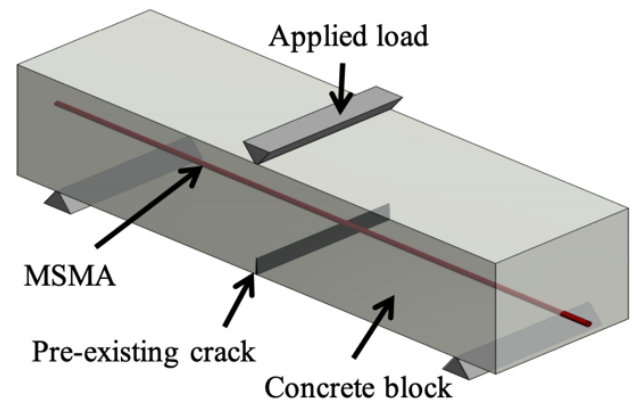

Fig. 1. The structural model consists of an embedded MSMA wire in a concrete mortar block.
For proper predictive damage detection and modeling of concrete, crack propagation, and SMA phase transformation due to stress, cohesive bonding between the concrete and wire and the fracture mechanics must be modeled in conjunction with one another. This is achieved by correlating the computational model to experimental testing by recreating experimental testing in the computational framework

The finite element model uses a traction-separation model to calculate the fracture. This model uses a scalar damage parameter $\mathrm{D}$, which ranges from $0-1$. This model requires the fracture energy of the material, which is derived from literature and experimental testing. Experimental testing was conducted on the concrete block and wire system in the manner previously described. The displacement-controlled testing showed a close relation between experimental testing and computational modeling to the point of fracture, crack propagation, and loading on the MSMA to induce phase transformation.

The finite element model utilizes a User Material Subroutine (UMAT) to characterize the shape memory alloy behavior present in the wire, determining the resulting martensite volume fraction of the MSMA wire. The foundation and background for the constitutive model for the SMA structural response is provided elsewhere [12] and has been used to analyze a number of SMA applications [13, 14].

\subsection{Magneto-static modeling}

After the structural state of the damaged specimen is determined structurally, the effectiveness of magnetic sensing is evaluated and simulated using COMSOL Multiphysics (a cross-platform finite element software to solve coupled systems of partial differential equations). This study investigates the sensing capabilities of an external magnet and sensor for the purpose of detecting the internal structural damage using the change in the external magnetic field.

The magneto-static computational model utilizes Ampere's Law and Gauss's Law with the assumption of zero applied currents,

$$
\nabla \cdot B=-\nabla \cdot\left(\mu \nabla \phi_{m}-\mu M\right)=0
$$

where $\mathrm{B}=$ magnetic flux density, $\mu=$ material magnetic permeability, and $\mathrm{M}=$ magnetization field. The magnetic permeability $\mu=\mu_{o} \mu_{m}$, where $\mu_{o}=$ the permeability of free space and $\mu_{m}=$ the material relative permeability. The material relative permeability of austenite and martensite are different, which means a change in the phase of the MSMA will change the magnetic boundary value problem and measured external magnetic flux.

The model, shown in Figure 2, consists of an MSMA wire embedded in a concrete block, with a permanent magnet on one side of the concrete block generating a magnetic field. The permanent magnet is defined by its residual flux density of $1.3 \mathrm{~T}$, the strength of the magnet used in material calibrations and a common strength for commercial AlNiCo and Neodymium N42 magnets. The 
magnetic properties of the MSMA wire are taken from magnetic testing calibration data as shown in Figure 3. The relation between magnetic flux density and magnetic field strength is linear below the saturation flux density value of $\sim 0.5 \mathrm{~T}$ and determined by the material magnetic permeabilities.

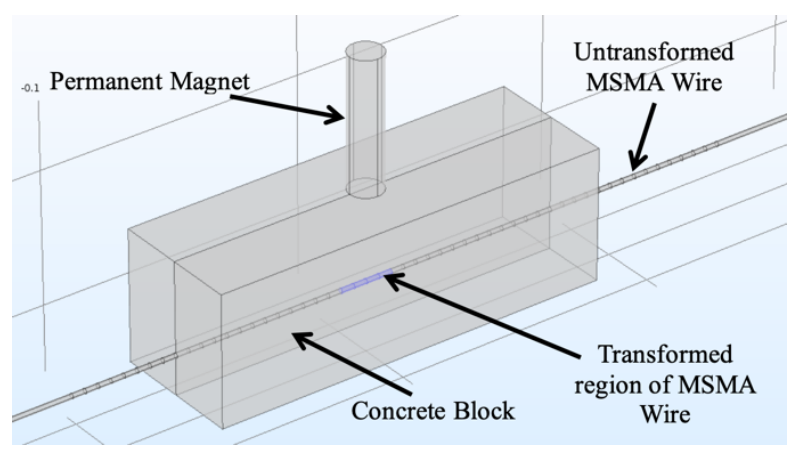

Fig. 2. The magneto-static model consists of a wire with a transformed portion at the center of the concrete block with a magnet placed on one side of the block.

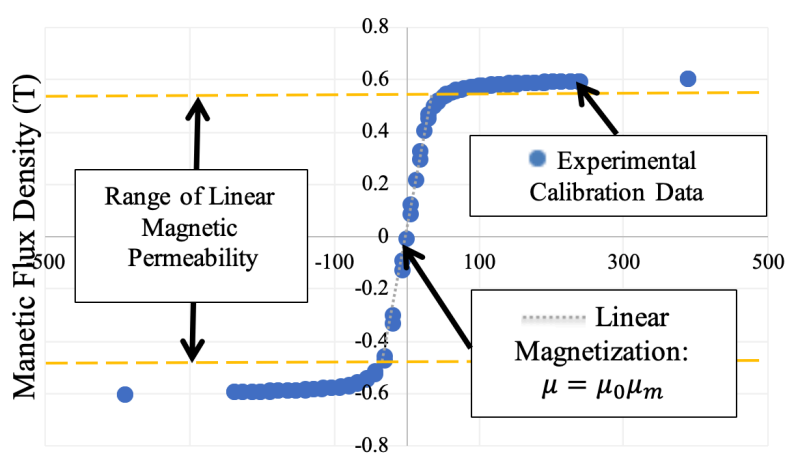

Magnetic Field Strength $\left(\frac{A}{m} * 10^{4}\right)$

Fig. 3. The magneto-static model relies on a linear relation between magnetic flux density and magnetic field strength, known as the relative magnetic permeability.

The magnetic field normal flux is computationally calculated for a magnet with known parameters and a probe moving axially away from center of the magnet's face. These results are then compared to experimental results for the same parameters and a known analytical solution for this simple geometry to ensure the parameters, computational model, and scripting interface are in agreement, as shown in Figure 4.

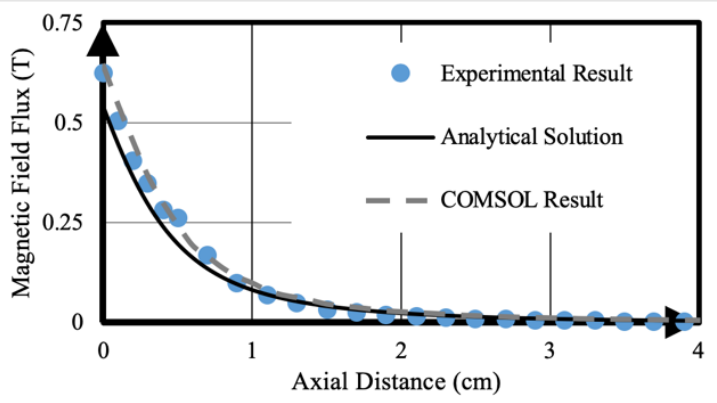

Fig. 4. A comparison between computational results obtained from the magneto-static model, experimental results, and analytical solutions for a simple geometry.
For a steady-state model, the formation of stressinduced martensite (SIM) can be correlated to a change in the measured magnetic flux density at an external location. This serves as a method to locate and detect internal cracks and damage inside the concrete block.

The region of SIM creates a disturbance in the magnetic field generated by the permanent magnet, and a search is simulated by moving a magnet and sensor along the face of the structure parallel to the embedded wire, as shown in Figure 5. The distance between the magnet and sensor is held constant, and the wire is embedded $15 \mathrm{~mm}$ away from the top face of the concrete block.

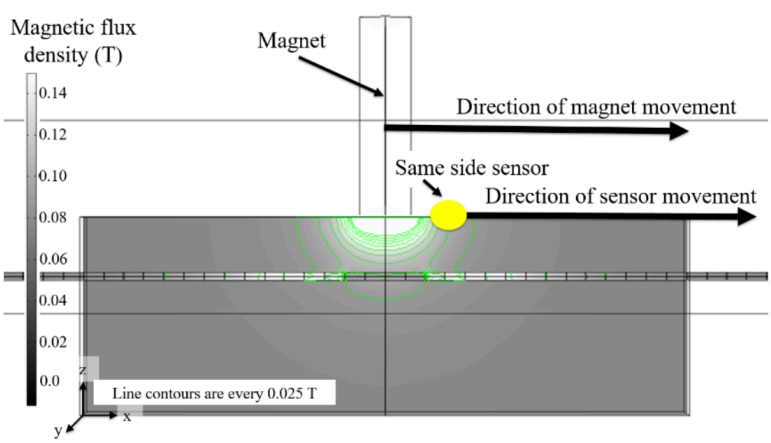

Fig. 5. The sensor and magnet move along the length of the concrete block measuring the external magnetic flux in the vicinity of stress-induced martensite (SIM), with the sensor 15 $\mathrm{mm}$ away from the wire. The change in measured magnetic flux by the sensor indicates the presence of SIM, indicating the local increase in stress near an internal crack.

\section{Results}

This section will lay out the results found by using the previously mentioned methods for structural and magnetic evaluation. First the structural results determine the change in martensite volume fraction in the MSMA wire. The change in martensite volume fraction is then input into the magneto-static model to calculate the change in an external, measured magnetic field. This magnetic flux profile is then used to determine the presence and location of internal cracks near the wire.

\subsection{Structural results}

The structural model evaluates the state of the MSMA wire as a crack propagates through the concrete block. The load applied to the concrete is transferred to the wire, particularly near the crack. The crack tip stress concentration and crack propagation transform portions of the nearby MSMA wire from austenite into SIM, as shown in Figure 6.

The model displays a strong local increase in stress and SIM near the crack tip, as to be expected for a crack tip field. The cohesion between the wire and the concrete breaks near the areas of higher stress, due to the differences in thermo-elastic properties between FeMnAlNi and concrete. The result is an increase in martensite volume fraction near the damage as illustrated above. 


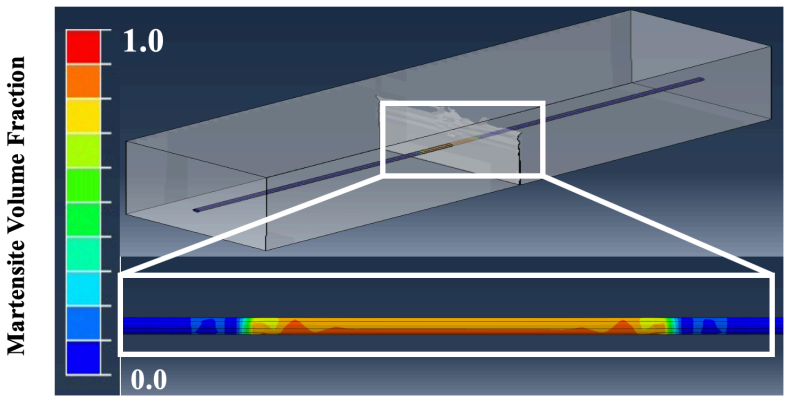

Fig. 6. The martensite volume fraction of the MSMA wire increases around the propagated crack. The concrete's failure leads to increased loading on the wire centered on the concrete failure.

\subsection{Magnetic sensing results}

With the change in martensite volume fraction determined from the structural computational results for a nearby embedded crack, the magneto-static model inputs the MSMA wire with a $2 \mathrm{~cm}$ transformed length of wire at its center. A magnet and sensor are moved down the length of the wire on the same side of concrete block to measure the change in magnetic flux near the crack. These scripted search results are shown in Figure 6 , for distances between the magnet and sensor of both 25 and $50 \mathrm{~mm}$. The change in magnetic flux near the crack tip is due to the formation of SIM near the crack tip, and leads to a measurable change in flux density.

While comparing individual data points is useful, a more robust technique for evaluating the viability of same side sensing locations and the effect caused by the phase transformation's magnetic disturbance is to observe the change between far field magnetic flux and the magnetic flux near the SIM region for a complete grid of locations, since the evaluations are done in postprocessing. This requires looking at the entire field and its changes in a single plane, the plane passing through the concrete block's top face, at different distances from the crack tip, as shown in Figure 7. In this figure, the change in magnetic flux due to the SIM region is shown for all possible sensor locations on the top face of the structure.

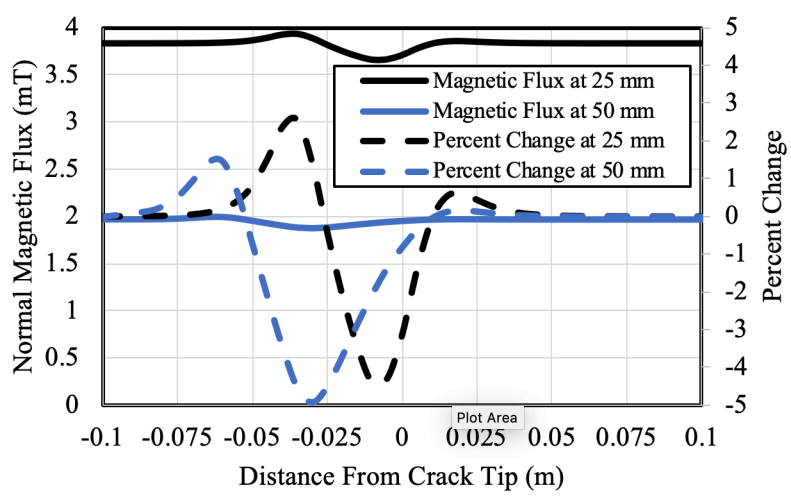

Fig. 7. The change in magnetic flux measured increases as the sensor passes by the local wire transformation shows a distinct pattern, which is dependent on the distance between the sensor and magnet location in relation to the transformed wire.

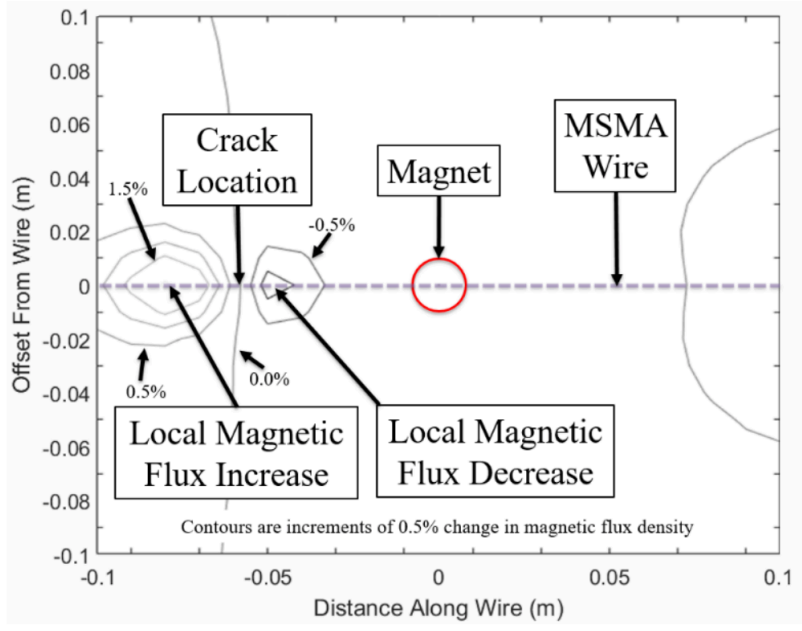

Fig. 8. An evaluation of all possible sensor locations for same side sensing reveals how the magnetic flux density increases near the crack on one side, but decreases on the other. The largest percent changes in magnetic flux density also occur along the length of the wire.

\section{Conclusions}

The primary purpose of the framework is to evaluate the feasibility of MSMAs (specifically FeMnAlNi) as multifunctional sensors for SHM using NDE. In this work, a novel technique is presented for non-destructive evaluation via embedded MSMA wires using a coupled structural and magneto-static evaluation to determine the effect of stress-induced martensite in an MSMA wire near an internal crack on the local and global magnetic field created by a permanent magnet. The structural framework presented demonstrates the transformation of a FeMnAlNi wire under loading near a propagating internal crack leading to the formation of stress-induced martensite, which can be correlated to the change in the wire's magnetic permeability. The subsequent magnetostatic sensing studies investigate the change in magnetic flux density near the stress-induced martensite region, and the capability to sense the internal damage using an external magnet and sensor.

As the permanent magnet and sensor are moved down the length of wire along the same side of the concrete block, the measured magnetic flux changes near the MSMA wire's phase transformation. For the single wire in a structural model, there is significant change in the magnetic flux near the crack. The magnet and sensor location combined with the magnetic flux profile along the MSMA wire provide evidence of internal cracks and damage near the wire under large loading. The presented novel framework and initial results presented support the feasibility of structural health monitoring using magnetic shape memory alloys for magnetic sensing.

\section{References}

1. Mirsayar, M.M., Razmi, A., Berto, F., n.d. Tangential strain-based criteria for mixed-mode I/II fracture toughness of cement concrete. Fatigue 
Fract. Eng. Mater. Struct. 41, 129-137. https://doi.org/10.1111/ffe.12665

2. Razmi, A., Mirsayar, M.M., (2017). On the mixed mode I/II fracture properties of jute fiber-reinforced concrete. Constr. Build. Mater. 148, 512-520. https://doi.org/10.1016/j.conbuildmat.2017.05.034

3. Shi, X., Mirsayar, M., Mukhopadhyay, A., Zollinger, D. (2019). Characterization of twoparameter fracture properties of portland cement concrete containing reclaimed asphalt pavement aggregates by semicircular bending specimens. Cem. Concr. Compos. 95, 56-69. https://doi.org/10.1016/j.cemconcomp.2018.10.013

4. Zafar, A., Andrawes, B. (2013). Experimental flexural behavior of SMA-FRP reinforced concrete beam. Front. Struct. Civ. Eng. 7, 341-355. https://doi.org/10.1007/s11709-013-0221-y

5. Tseng, L.W., Ma, J., Wang, S.J., Karaman, I., Kaya, M., Luo, Z.P., Chumlyakov, Y.I. (2015). Superelastic response of a single crystalline FeMnAlNi shape memory alloy under tension and compression. Acta Mater. 89, 374-383. https://doi.org/10.1016/j.actamat.2015.01.009

6. Monroe, J.A., Karaman, I., Basaran, B., Ito, W., Umetsu, R.Y., Kainuma, R., Koyama, K., Chumlyakov, Y.I. (2012). Direct measurement of large reversible magnetic-field-induced strain in $\mathrm{Ni}-$ Co-Mn-In metamagnetic shape memory alloys. Acta Mater. 60, 6883-6891. https://doi.org/10.1016/j.actamat.2012.07.040

7. Simonen, J.T., Andringa, M.M., Grizzle, K.M., Wood, S.L., Neikirk, D.P. (2004). Wireless sensors for monitoring corrosion in reinforced concrete members. Presented at the Smart Structures and Materials 2004: Sensors and Smart Structures Technologies for Civil, Mechanical, and Aerospace Systems, International Society for Optics and Photonics, 587-597. https://doi.org/10.1117/12.539529

8. Popovics, J.S., Gallo, G.E., Shelton, M., Chapman, P.L. (2007). A magnetic sensing approach to characterize corrosion in reinforced concrete. Presented at the Sensors and Smart Structures Technologies for Civil, Mechanical, and Aerospace Systems 2007, International Society for Optics and Photonics, p. 65291A. https://doi.org/10.1117/12.714307

9. Bielefeldt, B.R., Benzerga, A.A., Hartl, D.J. (2016). Analysis of shape memory alloy sensory particles for damage detection via substructure and continuum damage modeling. Presented at the Behavior and Mechanics of Multifunctional Materials and Composites 2016, International Society for Optics and Photonics, p. 98000B. https://doi.org/10.1117/12.2219260

10. Choi, E., Mohammadzadeh, B., Kim, D., Jeon, J.-S. (2018). A new experimental investigation into the effects of reinforcing mortar beams with superelastic SMA fibers on controlling and closing cracks. Compos. Part B Eng. 137, 140-152. https://doi.org/10.1016/j.compositesb.2017.11.017
11. Zheng, B., Dawood, M. (2017). Fatigue crack growth analysis of steel elements reinforced with shape memory alloy (SMA)/fiber reinforced polymer (FRP) composite patches. Compos. Struct. 164, 158-169. https://doi.org/10.1016/j.compstruct.2016.12.077

12. Lagoudas, D., Hartl, D., Chemisky, Y., Machado, L., Popov, P. (2012). Constitutive model for the numerical analysis of phase transformation in polycrystalline shape memory alloys. Int. J. Plast. 32-33, $155-183$. https://doi.org/10.1016/j.ijplas.2011.10.009

13. Hartl, D.J., Chatzigeorgiou, G., Lagoudas, D.C. (2010). Three-dimensional modeling and numerical analysis of rate-dependent irrecoverable deformation in shape memory alloys. Int. J. Plast. 26, $1485-1507$. https://doi.org/10.1016/j.ijplas.2010.01.002

14. Peraza Hernandez, E.A., Hu, S., Kung, H.W., Hartl, D., Akleman, E. (2013). Towards building smart self-folding structures. Comput. Graph., Shape Modeling International (SMI) Conference 2013 37, 730-742. https://doi.org/10.1016/j.cag.2013.05.022 